\title{
Ueber die Complication des Puerperiums mit chronischen Herzkrankheiten.
}

Von

Otto Spiegelberg.

I. Wenn eine mit einem Herzfehler behaftete Frau schwanger wird, so können schon in der Schwangerschaft wie besonders auch nach der Entbindung die übelsten Zufälle entstehen, ja es kann durch den puerperalen Zustand das tödtliche Ende rasch herbeigeführt werden. - Wenn ich von der gelegentlichen Anführung hieher gehöriger Fälle in den Jahresberichten der verschiedenen Gebäranstalten und von der Aufstellung der in Rede stehenden Complication als einer eventuellen Indication zur künstlichen Fruihgeburt absehe, so finde ich diesen übelen Einfluss des Puerperiums nur von Hecker im 1. Bande seiner ,Klinik der Geburtskunde“ (Seite 173) unter Mittheilung zweier Krankheitsfälle erörtert. Da die Complication, wie sich schon a priori erwarten lässt, keine gerade seltene ist, so will ich einige meiner einschlägigen Beobachtungen deshalb hier mittheilen, weil ich die nachtheilige und, wie bemerkt, bisweilen deletäre Wirkung der Schwangerschaft und des Wochenbettes auf die Klappenfehler bestimmter auf ihre wahren Ursachen zurückführen zu können glaube, als dies von Hecker geschehen ist.

H. findet die Gefahr, welche für der Art kranke Schwangere gesetzt wird, bedingt ,einmal durch die in den letzten Monaten stattfindende Beengung des Thoraxraumes, wodurch die durch den 
Herzfehler in ihrer Function schon beeinträchtigten Lungen mitunter ganz acut durch eine seröse Ausschwitzung functionsunfähig werden; oder es wird das Hèrz durch die Anstrengungen bei der Geburt so in Anspruch genommen, dass sein schon gestörter Mechanismus völlig in Verwirrung kommt und dadurch dem Leben ein Ende gemacht wird".

So richtig dies im Allgemeinen ist, so umschreiben obige Sätze doch mehr eine richtig beobachtete Thatsache, als sie dieselbe erklären; auch werden die Fälle, in welchen der in der Gravidität entwickelten Gefahr durch die Geburt ein Ende gemacht wird, und die in denen die Gefahrerst nach der letzteren auftritt, durch sie nicht erläutert.

Diese übelen Zufälle werden vielmehr nur dadurch bedingt, dass der für gewöhnliche Verhältnisse zu einem erträglichen Zustande genügende Grad der Compensation des Herzfehlers für veränderte und gar nun für plötzlich veränderte Druckverhältnisse nicht mehr ausreicht; in dieser Beziehung ist aber in erster Linie nicht die - abgesehen von ungebürlicher Ausdehnung des Abdomens ganz unwesentliche, wenn überhaupt vorhandene '), Beschränkung des Thoraxraumes bei Schwangeren von Bedeutung, sondern der veränderte Druck, unter welchem das Herz in der Schwangerschaft resp. nach der Geburt arbeitet.

Durch die Einschaltung des.Placentarstromgebietes zwischen Uterinarterien und - venen, durch die, wenn auch experimentell noch nicht festgestellte, aber klinisch unabweisbare, Zunahme des Blutquantums in der Schwangerschaft steigt der Widerstand im Gebiete der Aorta und damit die Arbeit für das Herz. Dem entsprechend nimmt letzteres nach den übereinstimmenden und in ihren Resultaten nicht anzuzweifelnden Beobachtungen von Larcher, Ducrest, Beraud, Blot²) und Duro$z i e z^{3}$ ) in seiner linken Hälfte an Masse zu, es entsteht Hypertrophie des linken Ventrikels. - Mit der Ausstossung des Kindes und seiner Anhänge ändern sich diese Verhältnisse rasch, der Placentarkreislauf wird eliminirt, die zahlreichen und weiten Utero-

1) cf. Dohrn in Monatsschr. f. Geburtsk. XXIV. S. 414, u. Gusserow, ibid. XXXII. S. 88.

2) cf. Joulin "Traité complet d'Accouchements". 1867. I. S. 383.

3) Gaz. des Hôpit. 1868. Nr. 104. 
Placentargefässe geschlossen - der Druck in der Aorta sinkt; steigt hierdurch schon der Druck im venösen Stromgebiete, so geschieht dies noch mehr deshalb, weil bei zum Theil unwegsam gewordenen Bahnen (im Uterus) die Blutmasse doch immer noch relativ vermehrt ist; denn der Blutverlust bei der Geburt ist in normalen Fällen nicht so reichlich, um die vorher vorhandene Vermehrung der Masse sofort auf die nichtpuerperale Norm zu reduciren. Zufolge der so entstandenen Drucksteigerung in den Venen, $z u$ der in demselben Sinne wirkend noch die durch die Entleerung der Bauchhöhle eingetretene grössere Excursionsfähigkeit des Zwerchfells tritt - strömt nach der Entbindung reichlicher Blut in die Brusthöhle und zu den Lungen; die Arbeit für das rechte Herz wächst.

Es ist nun leicht verständlich, dass diese von einem gesunden Herzen bald überwundenen ủnd ausgeglichenen Druckänderungen auf ein in seiner Mechanik durch einen Klappenfehler gestörtes turbirend einwirken können; wann und wie weit sie dies thun, wird wesentlich abhängen von dem Sitze des Fehlers, und davon, ob er ein compensirter oder ob er noch ein relativ frischer ist, bei dem es noch nicht zur Compensation der Störung gekommen. Dabei können Nebenumstände, wie der Grad der Ausdehnung des Bauches in der Schwangerschaft, also der der Excursionsfähigkeit des Zwerchfells, das Quantum des Blutverlustes in und gleich nach der Geburt, immer noch modificirend auf die Erscheinungen influiren.

II. Bei der Insufficienz der Aortenklappen 1) treten in der Regel schon in der Schwangerschaft, gewöhnlich in der 2. Hälfte derselben, bedrohliche Störungen der Körper- und Lungencirculation ein, weil so häufig die Compensation des Fehlers für die rasch steigenden Widerstände im arteriellen Gebiete nicht genügt; noch schlimmer wird es, wenn der Fehler noch jungen Datum's. Jene functionellen Störungen, besonders die dyspnoëtischen Anfälle und die starke unregelmässige Herzaction, führen nicht selten zu vorzeitiger Unterbrechung der Gravidität; in der Geburt erreichen die Erscheinungen die bedrohlichste Höhe; mit der Vollendung derselben aber tritt, weil der Druck in den Venen

1) Von den Fenlern des rechten Herzens sehe ich ab, da mir darüber keine Erfahrungen zur Disposition stehen, kann es bei deren Seltenheit auch. 
nun plötzlich steigt, der in der Aorta sinkt, Remission und oft ebenso plötzliche „Genesung“ ein.

Folgende Fälle dienen zur Illustration:

E. G. - Nr. 243. $186^{6} / r-34$ Jahre alt, hat vor 6 Jahren eine normale Schwangerschaft und Geburt überstanden; seit zwei Jahren leidet sie an Herzklopfen und Athemnoth. Sie befindet sich in der 27. - 28. Woche schwanger (17. April) und klagt über heftige Palpitationen, mit Präkordialangst und bedeutender Dyspnoe, wenn sie sich in horizontaler Lage befindet; die erste Schwangerschaftshälfte war ohne erhebliche Beschwerden verlaufen. - Patientin sieht bleich und gedunsen aus, Lippen eyanotisch, Augen prominirend und von starkem Glanze, Conjunctivae injicirt, die klopfenden Carotiden vibriren unter dem Finger, in der Foss. jugul. sieht und fühlt man Pulsation; der Radialpuls (90) ist resistent, hüpfend mit hoher kurzer Welle, sein Rythmus regelmässig. Die Lungen zeigen ausser etwas Bronchialkatarrh normale Verhältnisse, dagegen fällt die diffuse $\mathrm{Er}$ schïtterung der linken Thoraxwand, die Pulsation im Epigastrium und die systolische Erhebung in der Ausdehnung des linken Ventrikels auf. Der abnorm resistente Spitzenstoss ist über den 6 . und 7 . Intercostalraum verbreitert und ïberragt die Mammillarlinie um $3 \mathrm{Cm}$. nach links; am linken Sternalrande unterhalb der 4. Rippe deutliches Fremissement; die an der 3. Rippe beginnende Herzdämpfung erreicht im 7. Intercostalraume ihre grösste Breite, weit nach links uiber die Mammillarlinie hinaus; die übrigen Grenzen sind normal, so dass die Umfangszunahme ausschliesslich dem linken Ventrikel zukommt und eine Vergrösserung des Herzens hauptsächlich in der Längsrichtung stattfindet. - An der Herzspitze ist der laute erste Ton von einem schwachen Strepitus, der zweite von einem stark blasendeu Aftergeräusche begleitet; beide Geräusche werden an der Herzbasis intensiver und sind am deutlichsten am linken Sternalrande in der Höhe der 4. Rippe. Im 2. Intexcostalraume an der rechten Sternalseite sind sie weniger deutlich, dagegen der zweite Ton klingender, höher und verstärkt. Die Töne der Pulmonalis sind rein. An der Carotis systolisches Blasen und lauter diastolischer Ton. Leber in geringem Grade vergrössert, Füsse leicht ödematös, Harn mit Spuren von Eiweiss.

Hiernach war also Insufficienz der Aortenklappen evident, höchst wahrscheinlich ohne Stenose - weil für letztere der Puls zu voll und resistent, die Spannung im Aortensysteme zu hoch und weil die Erscheinungen der Rückstauung in die Venen zu wenig ausgesprochen; der an der Herzbasis am deutlichsten hörbare Strepitus war walrscheinlich durch Rauhigkeiten an der dem Blutstrome zugekehrten Fläche der Klappen bedingt. Dass dagegen die abnorm hohe Spannung im arteriellen Systeme nur auf Rechnung abnormer Widerstände an der Peripherie zu setzen. bewies die Verstärkung des zweiten Aortentones, welche Erscheinung bei Insufficienz oder Stenose des Aortenostium's sonst nicht vorkommen kann; ebenso wie 
das Fehlen abnormer Töne in den kleinen Arterien, welche der Aorteninsufficienz sonst eigenthümlich sind, aber nur bei normaler oder geringer Spannung der Arterien, nie bei erhöhter zu Stande kommen.

Unter Verabreichung salinischer Purgantien, ruhigem Verhalten und leichter Diät minderten sich die Stauungserscheinungen in den Lungen und im grossen Kreislaufe etwas, traten aber bei Wiederaufnahme der gewohnten Beschäftigung in verstärktem Maasse hervor und führten nach cirea sechs Wochen zur Unterbrechung der Schwangerschaft (3. VI.), in ungefähr der 34. Woche. Die Geburt verlief glïcklicherweise rasch, besonderer Anstrengungen bedurfte es nicht bei der kleinen Frucht, die uibrigens erhalten blieb. Mit der Entbindung traten alle bedrohlichen Erscheinungen rasch zurück und schwanden unter Purgiren, reichlichen Schweissen und starker Urinsecretion rasch. Am 8. Tage des Wochenbettes verliess die G., anscheinend ganz gesund, die Klinik.

Aehnlich, aber weniger stürmisch, verlief der folgende Fall, den ich kurz referire; die Insufficienz war alten Datum's.

Die 19jährige Nähterin Th. S., eine kräftige grosse Blondine, suchte im September 1868 Hülfe in der Poliklinik. Als Kind hatte sie, in feuchter Wohnung lebend, viel an rheumatischen Affectionen gelitten, deren Spuren noch an den Gelenken sichtbar; seit langer Zeit hatte sie Herzklopfen und Kurzathmigkeit bei Körperanstrengungen, ihrem ruhigen Berufe aber kann sie ungestört nachgehen. Im April will sie schwanger geworden sein. Sie klagte bei der Vorstellung über ein seit Wochen bestehendes verstärktes Herzklopfen und heftiges Pochen im Kopfe, das ihr die Nachtruhe raubte; vor acht Tagen hatte sie Nachts einen Anfall von Angina pectoris; die Nacht vor der Meldung hatte derselbe sich wiederholt. - Wir mussten sie sechs Monate schwanger schätzen. Sie zeigte sehr geröthetes Gesicht, injicirte Augen, frequenten hüpfenden Puls, nur wenig beschleunigte Respiration, keine Cyanose, kein Oedem, eiweissfreien Urin; Lungen frei, aber Aorteninsufficienz mit bedeutender $\mathrm{Hy}_{-}$ pertrophie namentlich des linken Ventrikels. - Die Behandlung war dieselbe, wie im vorigen Falle; bei derselben fïhlte sich die Kranke freier, bei irgend welcher Anstrengung indess wiederholten sich obige Störungen. Wie wir vernahmen, ist Patientin nach sechs Wochen, also im achten Monate, unter Assistenz einer Hebamme leicht und rasch niedergekommen und hat schnell ein gutes Wochenbett durchgemacht.

III. Für die Störungen, welche die Insufficienz und meist gleichzeitige Stenose des linken Ostium atrioventriculare macht, ist noch viel mehr, als für die durch Fehler der Aortenklappen bedingten, die Ausdehnung des Bauches, also die Excursionsfähigkeit des Zwerchfelles, der Zustand der Lungen und besonders die vorhandene oder fehlende Compensa- 
tion durch Hypertrophie, hier des rechten Ventrikels, von Bedentung. Es sind dabei drei Fälle möglich:

1) Der Fehler verursacht gar keine beunruhigenden Erscheinungen. Dies ist der Fall, wenn die Unterleibsausdehnung gering, der Thoraxraum gar nicht beengt, wenn die Störung alten. Datum's und durch Massenzunahme des rechten Ventrikels ausgeglichen ist, und wenn durch entsprechenden Blutverlust in der Geburt die erhöhte Spannung beseitigt wird. So habe ich verschiedentlich derartige Kranke ganz unbeeinflusst durch das Puerperium gefunden.

2) Sind dagegen die Pulmonalbahnen durch den Herzfehler, also durch Rückstauung anhaltend überfüllt, so treten schon in der Gravidität, besonders in deren späteren Monaten, die Erscheinungen, gestörter Lungencirculation entweder allmälig oder unter stürmischen Symptomen plötzlich auf -- wenn aus irgend welcher Ursache das Zwerchfell stark in die Höhe gedrängt und in anhaltender Exspirationsstellung befindlich, dadurch die Abfïhrung des Blutes aus den Lungen in's Herz behindert und die respiratorische Flä.che rein mechanisch verkleinert ist. Bei ihrer allmäligen Entstehung können die Störungen zur entsprechend compensatorischen Hypertrophie des rechten Ventrikels führen, so dass sie hierdurch überwunden werden und nach erfolgter Geburt rascher Nachlass derselben, wie bei Aorteninsufficienz eintritt.

P. B., Nr. 151. 1867/8. - 30 Jahre alt, hat zwei Mal normal geboren und will immer gesund gewesen sein. Sie stellt sich am angeblichen Ende ihrer Schwangerschaft in der Klinik vor und klagt über einen seit Mitte derselben bestehenden quälenden Husten, über Athemnoth, die in der letzten Zeit so zugenommen, dass sie nur sitzend schlafen könne; auch sei der Bauch seit Kurzem stark gewachisen. - Sie sieht cyanotisch aus, hat sehr frequente oberflächliche Respiration, einen kleinen weichen und schnellen Puls, Oedem der Unterextremitäten und der Bauchdecken, starke Gefässerweiterungen auf letzteren, enorm durch Wasser ausgedehnten Uterus; etwas Ascites, aber eiweissfreien Urin. Die Untersuchung des Thorax zeigt die Leber bis zur fünften Rippe emporgedrängt, weit verbreitetes Schnurren und Pfeifen, emphysematöse Lungenränder und den Befund der Insufficienz und Stenose der linken O. atrio-ventr. mit geringer Hypertrophie und bedeutender Dilatation des rechten Herzens. - Unter Verabreichung kleiner Gaben der Digitalis und von Stimulantien liessen die subjectiven Beschwerden etwas nach; mit den einige. Tage nach der Aufnahme beginnenden Wehen aber stiegen 
die Brusterscheinungen zur bedenklichsten Höhe, welche eine V. S. erfordete, liessen indess mit dem Abgange des reichlichen Wassers rasch nach, und nach der Geburt trat volle Remission ein. Das Wochenbett verlief ungestört.

Entwickeln sich jene angegebenen Folgen aber rasch, so kann es plötzlich zu intensiver Dyspnoe mit Katarrh oder serösem Ergusse in die Lungen und von dort aus durch weitere Rückstaung zu allgemeinem Oedem, Eiweissharn, Ascites und selbst Pleuraerguss kommen, und das Leben höchst gefährdet sein. Solche Fälle hat gewiss jeder beschäftigte Geburtshelfer gesehen. Mit der die Schwangerschaft alsdann meist vorzeitig unterbrechenden Geburt erreichen die Symptome die grösste Höhe, lassen nach ihr nach und es stellt das gestörte Gleichgewicht sich bald wieder her; oder es geht der Sturm von Neuem los, wie die folgende Erörterung ergiebt.

3) Die Stauungserscheinungen in der Schwangerschaft können bei Mitralisfehlern noch jungen Datum's unter den sub 1) angeführten günstigen Verhältnissen ausbleiben, dafür aber bedrohliche Erscheinungen erst nach der Entbindung eintreten. Wie früher angegeben, wird mit der Geburt durch Abschneiden des grossen, dem placentaren Kreislaufe angehörigen Stromgebietes im Uterus und Umgebung eine bedeutende Drucksteigerung im venösen Systeme gesetzt; das rechte Herz kommt hierdurch plötzlich unter einen noch stärkeren Druck, als es in Folge der Insufficienz schon gestanden. Dem rechten Ventrikel wird mehr Blut zugeführt, er sucht dasselbe in die Lungenarterien zu entleeren, vom linken Herzen dagegen findet eine Rückstauung in die Lungenvenen statt. So kommt es nun zu turbulenter unregelmässiger Herzaction, zu seröser Ausschwitzung in die Lungen, zu heftiger Dyspnoe, zu mangelhafter Decarbonisation des Blutes; diese setzt die Herzenergie nur noch mehr herab - und so summiren sich die Erscheinungen gegenseitig. Manche der beschriebenen Fälle von schweren nervösen Herzstörungen im Wochenbette, von ,,cardiac apnoea" nach der Entbindung gehören in diese Kategorie. In sehr acut auftretenden Fällen kann rasch weit verbreitetes Lungenödem sich entwickeln und den Tod herbeiführen; in weniger rasch verlaufenden sind die Wirkungen der Rückstauung in die Körpervenen mit Bronchialkatarrh, heftigen dyspnoetischen Anfällen, die Symptome, welche unter entsprechender Behandlung, unter den im Wochenbette erfolgenden Ausscheidungen allmälig meist schwinden. 
Die gut gebaute, etwas bleich und gedunsen aussehende N. N., Nr. 146, 1866/7 - hatte unter guter Pflege nnd bei ruhigem Verhalten ihre Schwangerschaft gut ïberstanden und rechtzeitig ganz normal und schnell geboren; Kurzathmigkeit mit Herzklopfen bei schnellem Treppensteigen, Palpitationen bei Lage auf der linken Seite waren die einzigen Beschwerden in der letzten Schwangerschaftszeit gewesen. Bald nach der Entbindung fiel die Arythmie ihres nicht frequenten Pulses $(70)$, der in unregelmässigen Intervallen aussetzte, bei mittlerer Füllung und Spannung der Arterie auf. Nach einigen Stunden stellte sich Brustbeklemmung und Athemnoth ein, welche sich momentan bis zur Orthopnoe steigerte; mit vornübergebeugtem Oberkörper, die Hände iiber den flectirten Knien gefaltet, fand ich Pat. unter Anspannung aller respiratorischen Hiilfsmuskeln nach Athem ringend. Schon par distance waren feuchte kleinblasige Rasselgeräusche lıörbar; die Radialarterien eng und wenig gespannt, die Pulswelle klein und schwach, ungemein frequent $(140-150)$, das Gesicht cyanotisch. Die Thoraxuntersuchung zeigte beträchtliche Stauung in den Pulmonalgelässen und daraus resultirende seröse Transsudation in die Bronchien, abnorme Ausbreitung der Herzdämpfung nach rechts hin, den Spitzenstoss an normaler Stelle, lautes blasendes Aftergeräusch an der Spitze, das sich nach oben hin allmälig verliert. Das Abdomen frei; der Urin sparsam, hochroth, eiweisshaltig. - Nachdem der erste Sturm durch kleine aber häufige Dosen der Digitalis gemildert, ergab genauere Untersuchung intacte Lungenränder mit vesiculärem Athmen, Schnurren und Pf'ifen in den Bronchien, ausschliessliche Ausdehnung des Herzens nach rechts hin, den zwischen der 5. und 6. Rippe in der Mammillarlinie am stärksten zu fühlenden Spitzenstoss nach rechts verbreitert, über dem rechten Ventrikel vage Erschïtterung der Brustwand, deutliche Vibration in derselben Gegend; den ersten Ton an der Herzspitze von einem blasenden Aftergeräusche gedeckt, den zweiten dort auffallend schwach, in der Mitte des Sternum und rechts von ihm stärker werdend; links vom Sternum über der Pulmonalis die beiden Töne deutlich; den zweiten weder verstärkt noch klappend - Hypertrophie des rechten Ventrikels also nicht vorhanden.

Unter quälender Zunahme eines trockenen Hustens traten in den nächsten zwei Tagen wiederholt heftige Dyspnoe-Anfälle auf, welche unter andauerndem Gebrauche kleiner Digitalismengen, von Morphium und salinischen Abführmitteln allmälig schwanden. Am dritten Tage erschien der Puls voller, 110, Respiration 34, die Harnmenge hatte beträehtlich zugenommen, der Eiweissgehalt war sehr gering geworden; am vierten Tage Athmung ruliger und freier, 22, der Puls 80 , in unregelmässigen Intervallen wieder aussetzend; zählte man jedoch die Herzcontractionen nach dem ersten Herztone, so zeigte sich, dass auch während der Intermission des Radialpulses Herzeontraction stattfand, aber so schwach, dass die in die Aorta gepresste Blutwelle in der Radialis nicht mehr fühlbaren Effect hervorbrachte; im Uebrigen war der Puls voller, die Urinseeretion noch 
reichlicher und ganz eiweissfrei geworden. - Von nun an (täglich 10 ctgm. Digitalis) erfolgte stete Erholung, und Pat. konnte am 10. Tage entlassen werden.

In gleicher Weise wie eine nicht compensirte Mitralisinsufficienz, kann auch Emphysem der Lunge, überhaupt jede Erkrankung der letzteren, die in einer Verkleinerung der Respirationsfläche oder einer Blutüberfüllung besteht, durch die Geburt und die mit ihr einhergehende Drucksteigerung im venösen Systeme influirt werden - wenn eben die Lungen nicht im Stande sind, plötzlich ein grösseres Quantum Blut aufzunehmen und durchzuleiten.

IV. Aus den vorhergehenden Mittheilungen ergiebt sich nun auch für die Behandlung der in Rede stehenden Complication. die wichtige Folgerung, dass dieselbe bei Fehlern der Aortenklappen eine wesentlich andere sein muss, als bei denen der Mitralis und besonders bei den nicht compensirten Fehlern dieser. Bei den ersteren darf von der Digitalis - welche so gewöhnlich bei allen Herzfehlern pêle-mêle gegeben wird - kein Gebrauch gemacht werden, weil kleine Dosen den Seitendruck in den Gefässen erhöhen, somit die Beschwerden steigern, grössere Gaben aber die Herzenergie herabsetzen und hiermit dem so günstigen compensatorischen Momente der Hypertrophie des linken Ventrikels entgegenarbeiten werden. Unter diesen Umständen ist nur ein ruhiges Verhalten, kühlendes Getränk, besonders aber starkes Purgiren mittels der Salina, bei sehr schweren Störungen eine reichliche V. S. indicirt.

Dass unter letzteren Umständen die künstliche Frühgeburt am Platze, liegt ebenfalls auf der Hand; mit der Entleerung des Uterus sinkt ja der Druck in der Aorta, und die obigen Beobachtungen zeigen, dass die Schwangerschaft nicht selten auch spontan unterbrochen wird. - Ebenso kann diese Operation bei Fehlern der Mitralis gerechtfertigt, ja nothwendig erscheinen, wenn die sub III. 2. angeführten Verhältnisse vorliegen. Niemals aber darf sie bei nicht compensirter noch frischer Mitralisinsufficienz ausgeführt werden, wie überall nicht da, wo eben die durch die Geburt gesetzte Drucksteigerung und Lungenüberfüllung zu fürchten ist. Für die Pneumonie ist dies schon von Gusserow ${ }^{1}$ ) klar dargethan. - In solchen Fällen ist vielmehr

1) 1. c. S. 87-94. 
tonisirend auf die Herzaction zu wirken, und durch Erregung der muskulomotorischen Nerven die Spannung und Füllung im Aortensysteme zu erhöhen, andererseits durch Erregung des Vagus, des regulatorischen Systemes die Pulsfrequenz herabzusetzen und somit auch die abnorme Füllung im Venensysteme zu vermindern. Beiden Zwecken entspricht die Digitalis in kleinen Dosen; dem letzteren besonders die V.S., welche unter bewandten Umständen wahrhaft lebensrettend werden kann. 